Sensors 2005, 5, 488-508

sensors

ISSN 1424-8220

(C) 2005 by MDPI

http://www.mdpi.org/sensors

\title{
Robust Multivariable Estimation of the Relevant Information Coming from a Wheel Speed Sensor and an Accelerometer Embedded in a Car under Performance Tests
}

\section{Wilmar Hernandez}

Department of Circuits and Systems in the EUIT de Telecomunicación at the Universidad Politécnica de Madrid (UPM), Campus Sur UPM, Ctra. Valencia km 7, Madrid 28031, Spain

Phone: +34913367830. Fax: +34913367829. E-mail: whernan@ics.upm.es

Received: 30 September 2005 / Accepted: 25 November 2005 / Published: 29 November 2005

\begin{abstract}
In the present paper, in order to estimate the response of both a wheel speed sensor and an accelerometer placed in a car under performance tests, robust and optimal multivariable estimation techniques are used. In this case, the disturbances and noises corrupting the relevant information coming from the sensors' outputs are so dangerous that their negative influence on the electrical systems impoverish the general performance of the car. In short, the solution to this problem is a safety related problem that deserves our full attention. Therefore, in order to diminish the negative effects of the disturbances and noises on the car's electrical and electromechanical systems, an optimum observer is used. The experimental results show a satisfactory improvement in the signal-to-noise ratio of the relevant signals and demonstrate the importance of the fusion of several intelligent sensor design techniques when designing the intelligent sensors that today’s cars need.
\end{abstract}

Keywords: wheel speed sensor; accelerometer; linear-quadratic optimal control; Kalman filter; linear-quadratic Gaussian control; loop transfer recovery.

\section{Introduction}

Due to the continually growing need for better comfort and safety in today's cars, a revolutionary way of designing sensors, actuators, and electrical and electromechanical systems for the automotive industry has been set. As a matter of fact, researchers all around the world have been encouraged to work not only on the fabrication of sensors but also on the fabrication of intelligent systems consisting of sensors, signal conditioners and microprocessors, among other devices, that incorporate a certain amount of intelligence into the sensors themselves. What is more, these signal conditioning and signal 
processing stages added to the sensors transform such sensors into robust and optimal measuring systems that are able to work satisfactorily in polluted, corrupted environments where we have little or no prior knowledge of either the relevant signal coming from the sensors or the noise corrupting their electrical information.

In short, one of the worst environments for sensors is that of the automotive industry. In the automotive industry sensors and electrical systems have to work under severe working conditions such as the endurance of high temperatures, high humidity, dangerous chemical attacks, undesirably strong vibrations, electromagnetic interferences, pollution, and so on [1-9].

Therefore, with this scenario in mind, no one would dispute that the robustness, optimality, efficiency and reliability of a system have been, and will continue to be, crucial.

The aim of the present paper is to design a robust and optimal multi-input multi-output system able to cancel noise and disturbances that corrupt the relevant information coming from both a wheel speed sensor and an accelerometer placed in a car under performance tests.

In this paper, our system is linear and its equations are in state space notation, where the system state vector consists of the speed and the acceleration of the car.

Section 2 concerns a general description of the principles of the sensors used in this paper. Section 3 concerns the design of the robust and optimal multi-input multi-output system. Section 4 concerns the results of the experiment. Section 5 concerns the conclusions.

\section{Sensors}

\subsection{Accelerometers}

\subsubsection{Principles}

In the industrial world, the most common design is based on a combination of Newton's law of mass acceleration and Hooke's law of spring action (Fig. 1) [10-26].

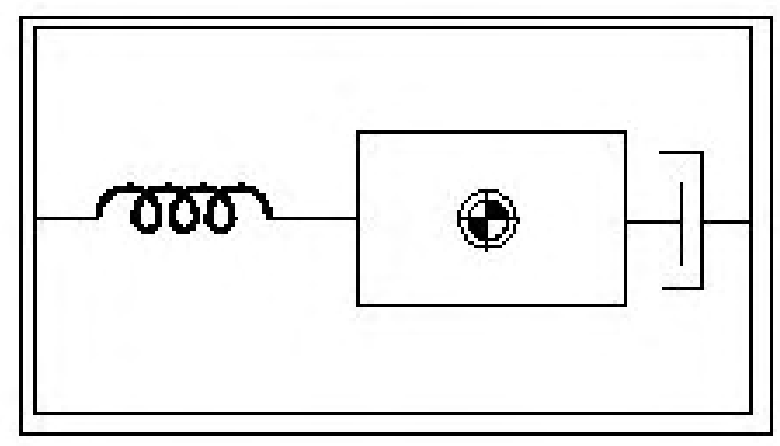

Figure 1. Basic spring-mass system accelerometer. 
According to Johnson [24], if the seismic mass $m$, is undergoing an acceleration $a$, then there must be a force $F$ acting on the mass and given by $F_{1}=m \cdot a$. In addition, the spring of spring constant $k$ is extended (or stretched) from its equilibrium position for a distance $\Delta x$ with a force $F_{2}$ (opposite to $F_{1}$ ) acting on the spring and given by $F_{2}=k \cdot \Delta x$. This condition is described by equating Newton's and Hooke's laws. Thus, under steady-state accelerations, the measurement of acceleration is reduced to a measurement of spring extension (linear displacement) (Eq. (1)).

$$
a=\frac{k}{m} \Delta x
$$

In analyzing the transient response, we should take into account the friction associated with the seismic mass. Furthermore, if the system exhibits oscillations, its frequency of oscillation is calculated as in Eq. (2), where $f_{N}$ is the natural frequency (Eq. (3)) and $\zeta$ is the dimensionless damping ratio.

$$
\begin{gathered}
f_{\text {osc }}=f_{N} \sqrt{1-\zeta^{2}} \\
f_{N}=\frac{1}{2 \pi} \sqrt{\frac{k}{m}}
\end{gathered}
$$

In this paper, it is considered that the movement of the vehicle's center of gravity can be discarded. Thus, the accelerometer is situated at the vehicle's center of gravity.

\subsubsection{Types of accelerometers}

There is a wide variety of accelerometers that could be used in different applications depending on the requirements of range, natural frequency, damping, temperature, size, weight, hysteresis, low noise, and so on. Piezoelectric accelerometers, piezoresistive accelerometers, variable capacitance accelerometers, linear variable differential transformers (LVDT), variable reluctance accelerometers, potentiometric accelerometers, gyroscopes used for sensing acceleration, strain gauges accelerometers, among others, are a part of the variety of accelerometers.

In this work, a variable capacitance accelerometer with range $\pm 2 g\left(g=9.81 \mathrm{~m} / \mathrm{s}^{2}\right)$ and sensitivity $985.6 \mathrm{mV} / \mathrm{g}$ at $5 \mathrm{~Hz}$ is used. Fig. 2 shows the frequency response of such a sensor (temperature $75^{\circ} \mathrm{F}$ and humidity 57\%).

\subsection{Wheel speed sensors}

The speed of rotation of the wheels is one of the most important inputs to the optimal braking system of the car. In addition, other uses of the information from the rotational speed of the car's wheel include: traction control, vehicle stability control, transmission control, engine management, chassis control, hill-holder brakes, rollback detection or electronic parking brakes, brake-force distribution and roll-over protection, among others.

At this point, it is important to point out that in spite of the fact that many rotational speed sensors share similar characteristics, not all of them are suitable for use in the automotive industry [6-27]. The reality is that angular motion sensors based on magnetic field sensing principles stand out because of 
their many inherent advantages and sensing benefits [9, 27]. In fact, angular motion sensors are the perfect choice across the whole automotive applications spectrum.

Amplitude Response

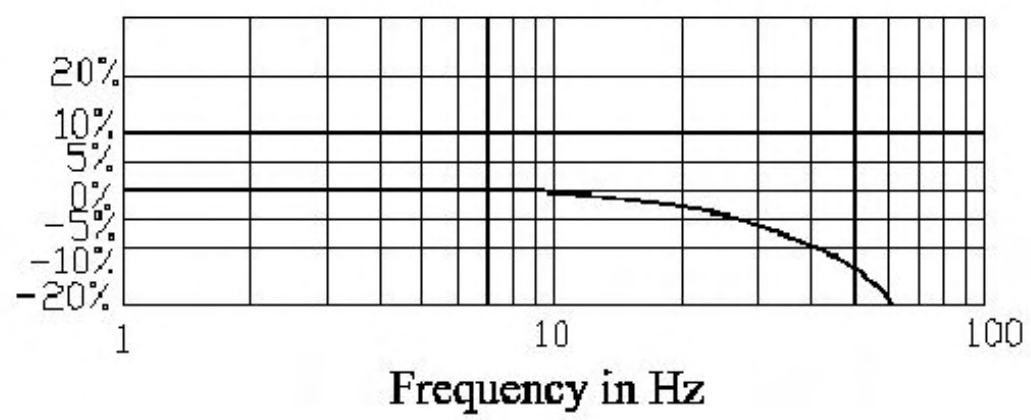

Figure 2. Frequency response of the accelerometer.

In this paper, a proximity sensor held in a protective casing and mounted in a fixed position close to one of the wheels of the car was used as the wheel speed sensor. The proximity sensor is of the variable reluctance type and its coil is consists of a thin wire wounded around an insulating form and coupled to a permanent magnet.

For the kind of tests carried out in this paper, this device was only used to measure the rotation of the wheels of the car; however, in the process-control industry this kind of sensor has many applications in measuring rotation, position and location.

\subsubsection{Principles}

When the proximity sensor detects the presence of any of the ferrous teeth of a toothed wheel, an output voltage is obtained (Fig. 3) because the ferrous teeth cross the magnetic field that is created in front of the sensor, causing a change in the resulting flow and producing an electromotive force in the coil. Thus, the output is an alternating signal whose frequency and amplitude are both proportional to the speed of rotation. A block diagram representing the measurement system is shown in Fig. 4.

\subsubsection{Considerations}

Due to the fact that proximity sensors are widely used in many industrial applications, it is important to say something about their advantages and disadvantages [9].

On the one hand, they can be very small and we put embed them in places where other sensors may not fit. In addition, they are often sealed in protective cases and can be resistant to high temperatures and high pressures as well as chemical attacks.

Other advantages are their reliability, the low maintenance required, and their low cost.

On the other hand, they have to be placed very close to a suitable ferrous metal to produce an adequate output voltage. They also suffer from undesirable signals or noise. In fact, these sensors have a very low signal-to-noise ratio at automobile speeds lower than $5 \mathrm{~km} / \mathrm{h}$ and give very corrupted and misleading information about the real speed of the car at speeds equal to or lower than the one 
previously mentioned. Such behavior is not appropriate for the braking performance. For this reason the anti-lock braking system (ABS) of most of today's cars is disconnected at the end of the braking process, and the car is finally braked but without the help of the electronic braking system.

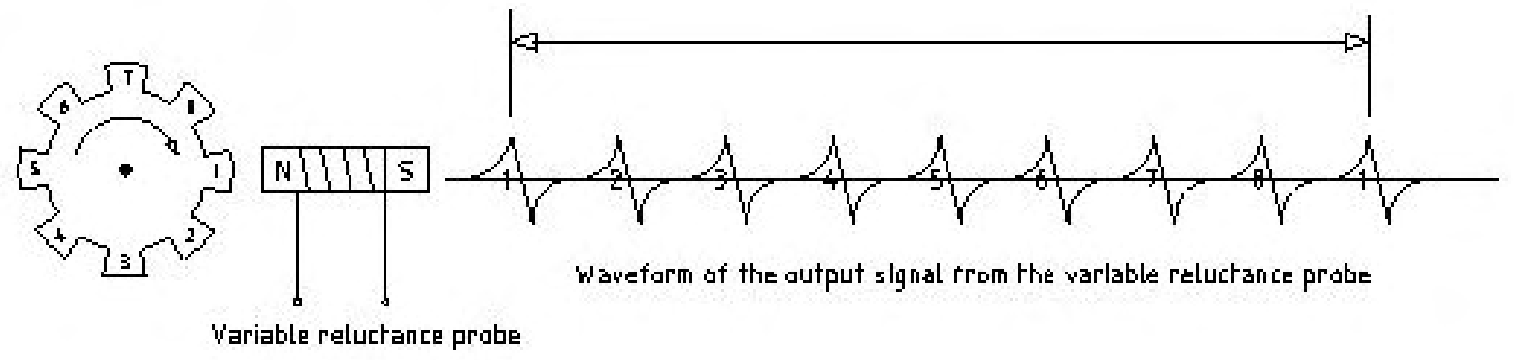

Figure 3. Wheel speed sensor.

Variable reluctance proximity sensor

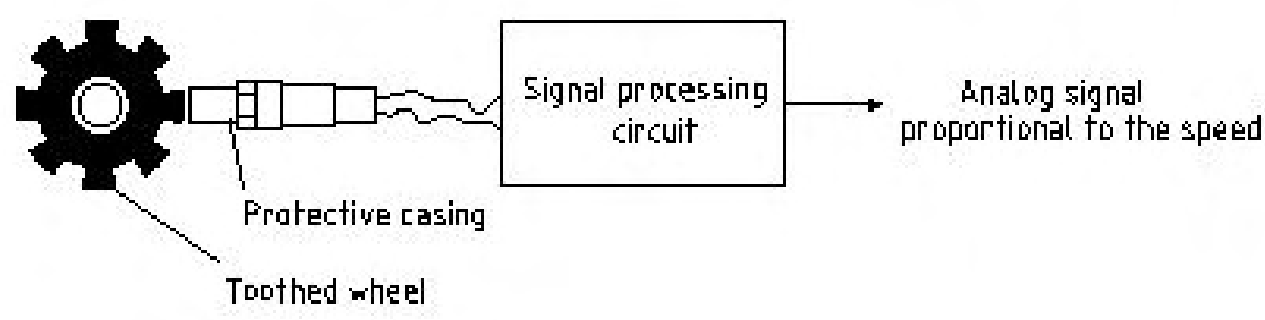

Figure 4. Block diagram of the measurement system. 
In summary, despite the fact that there are disadvantages, the variable reluctance proximity sensors seem to be the most suitable choice to measure the speed of rotation of motor car wheels in the ABS of today's automobiles. In this sense, these sensors play an important role in the optimal braking system of today's cars, which is a safety-related problem where effective and reliable performance has the highest priority.

\section{Design of the robust and optimal multi-input multi-output system}

\subsection{Introduction}

In the previous section both the accelerometer's and wheel speed sensor's principles were given. However, such sensors as shown in the fore mentioned section are not prepared to reject the undesirable effects of noise and disturbances corrupting their measurements. The reality is that the designer needs to deal with the consequences of the inevitable disturbances, noise and errors that cause sensor operations to deviate from their true value, which causes an undesirable degree of uncertainty in the measurements carried out by the sensors.

In short, due to the fact that some parameters of the structure of the models are uncertain, the designer is faced with structured or parametric uncertainties. This type of uncertainty negatively affects parameters that define the dynamics of the process such as mass, damping, natural frequency, and so on.

What is more, owing to the fact that the models are also in error because of missing dynamics, usually at high frequency, the designer is faced with neglected and not modeled dynamics uncertainties, which are present in every model of a real system.

This section focuses on the application of linear quadratic Gaussian control and loop transfer recovery (LQG/LTR) control techniques to shape the multi-input multi-output loop transfer function of the system, so that both the accelerometer and the wheel speed sensor can succeed in dealing with the inevitable sources of the above mentioned uncertainties.

At this point, it is important to point out that Kalman filtering and LQG/LTR control techniques are useful in applications in which we need to carry out the optimal observation of the state variables of feedback-controlled systems [28-60]. In addition, the optimal and robust controller obtained as a result of applying the LQG/LTR control technique does not have a high computational burden. What is more, such a controller has good numerical properties, and good transient and tracking performance. Furthermore, the LQG/LTR controller has good disturbance rejection and robustness.

On the other hand, LTR procedures have several disadvantages as well. For instance, their main disadvantage is that they are limited to minimum phase systems. In short, they should not be used to designing the controllers for non-minimum phase systems because the recovery procedures work by canceling the system zeros, which could lead to instability [28-60].

However, the multi-input multi-output sensor presented in this paper is a minimum phase system, and the results of the application of the LQG/LTR control technique were satisfactory.

\subsection{Modeling of the sensors as a multi-input multi-output dynamic system}

The multi-input multi-output dynamic system is given by Eq. (4) and Eq. (5) [28-60]. 


$$
\begin{aligned}
& \dot{x}(t)=A x(t)+B_{1}\left(u(t)+w_{2}(t)\right)+B_{2} w_{1}(t) \\
& y(t)=C x(t)+D u(t)+E v(t)
\end{aligned}
$$

In these equations, the plant under consideration is time-invariant and $A, B_{1}, C$ and $D$ are constant matrices and $B_{2}$ is a constant vector. $x(t)$ is the state vector, which consists of three components: the first component (that is, $x_{1}(t)$ ) is the displacement; and the second and third components (that is, $x_{2}(t)$ and $\left.x_{3}(t)\right)$ are the velocity and the acceleration, respectively.

In this paper, the velocity and the acceleration are estimated by using a Kalman filter (optimum observer). In addition, it is assumed that the acceleration is a Wiener process [61-63].

Furthermore, $u(t)$ is the plant input and $y(t)$ is the plant output. What is more, both $u(t)$ and $y(t)$ are vectors. $w_{1}(t)$ is white noise and represents process noise, and $w_{2}(t)$ is also white noise and represents extra process noise added directly to the control input. $v(t)$ is white noise as well and represents measurement noise [61-63].

Moreover, the process noise intensities have the same properties and are equal to $W$, which is in general a positive definite symmetric matrix. The measurement noise intensity is $V$, which is a positive definite symmetric matrix. $A, B_{1}, B_{2}, C, D$ and $E$ are given below (see Eq. (6) - Eq. (11)).

$$
\begin{aligned}
& A=\left[\begin{array}{lll}
0 & 1 & 0 \\
0 & 0 & 1 \\
0 & 0 & 0
\end{array}\right] \\
& B_{1}=\left[\begin{array}{ll}
1 & 0 \\
0 & 1 \\
0 & 0
\end{array}\right] \\
& B_{2}=\left[\begin{array}{l}
0 \\
0 \\
1
\end{array}\right] \\
& C=\left[\begin{array}{lll}
1 & 0 & 0 \\
0 & 1 & 0
\end{array}\right] \\
& E=\left[\begin{array}{ll}
1 & 0 \\
0 & 1
\end{array}\right]
\end{aligned}
$$

\subsection{The LQG/LTR method}

At this point, it is important to highlight that between the modern and post-modern control methods, the LQG/LTR control method is an outward one. It is focused on shaping the target feedback loop. 
However, there are others like the $H_{\infty}$ optimal control method where the designer of the controller shapes both the sensitivity function and the complementary sensitivity function [28-60, 64-67].

According to Doyle and Stein [33], the idea behind the LQG/LTR method is a complex one and it could be summarized as follows:

First of all, the attributes that the controller must have should be clearly established. To that end, the true design objectives should be expressed in mathematical terms as clear as possible. In short, a tradeoff should be established between what the designer wants and how the designer formulates it in mathematical terms.

In this method either the optimum observer is adjusted so that the loop transfer function can have a gain margin of $+\infty \mathrm{dB}$ and a minimum phase margin of $60^{\circ}$ for single-input single-output systems; or the optimum observer is adjusted so that the sensitivity function satisfies the condition that its maximum singular value is lower than or equal to 1 .

Secondly, a loop transfer function should be designed by using linear-quadratic optimal control methods so that the loop can have the desired margins mentioned above. In this step, the choice of the state-weighting matrix $Q$ and the control-weighting matrix $R$ depends on the kind of cost of the control that the designer wants to achieve and how the deviations of the state variables from the origin are going to be penalized. Consequently, a linear-quadratic optimal regulator (LQR) is obtained.

Thirdly, a Kalman filter should be designed so that the characteristics of the loop transfer function obtained in this step are similar to the ones obtained with the LQR designed in the previous step. In short, according to an adjusting procedure the Kalman filter is designed and shaped. The intensity of the extra process noise added directly to the control input of the plant is gradually increased and the more the intensity is increased, the better the robustness properties of the loop transfer function. In other words, the more the intensity of such a fictitious noise approaches infinity, the more the loop transfer function of the system recovers its robustness properties.

On the other hand, this ad-hoc design procedure of shaping the singular values of the loop transfer function could cause problems with unmodeled dynamics, because while it is carrying out the recovery of the robustness of the system, the gains get bigger step-by-step. This is why the recovery procedures are rarely carried out until their limits. Basically, the designers stop the process of recovering the robustness of the loop at the point where they consider that an acceptable design has been achieved.

\subsection{Design of the loop transfer function}

In accordance with the adopted approach in the previous subsections, Eq. (12) and Eq. (13) show the metasystem.

$$
\begin{aligned}
& {\left[\begin{array}{l}
\dot{x}(t) \\
\hat{x}(t)
\end{array}\right]=\left[\begin{array}{cc}
A & -B_{1} K_{C} \\
K_{O} C & A-B_{1} K_{C}-K_{O} C
\end{array}\right]\left[\begin{array}{l}
x(t) \\
\hat{x}(t)
\end{array}\right]+\left[\begin{array}{ccc}
B_{2} & B_{1} & 0 \\
0 & 0 & K_{O}
\end{array}\right]\left[\begin{array}{c}
w_{1}(t) \\
w_{2}(t) \\
v(t)
\end{array}\right]} \\
& {\left[\begin{array}{l}
y(t) \\
y(t)
\end{array}\right]=\left[\begin{array}{ll}
C & 0 \\
0 & C
\end{array}\right]\left[\begin{array}{l}
x(t) \\
x(t)
\end{array}\right]+\left[\begin{array}{ll}
D & E \\
0 & 0
\end{array}\right]\left[\begin{array}{l}
u(t) \\
v(t)
\end{array}\right]}
\end{aligned}
$$


where $K_{C}$ is the controller matrix gain and $K_{O}$ is the observer matrix gain. Furthermore, the procedure for the calculation of the previously mentioned matrices depends on solving the following equations

$$
\begin{aligned}
& A_{r}^{T} \Pi+\Pi A_{r}+Q-\Pi I B_{r} R^{-1} B_{r}^{T} \Pi=0 \\
& K_{L Q R}=R^{-1} B_{r}^{T} \Pi \\
& A \Theta+\Theta A^{T}+B_{2} W_{1} B_{2}^{T}+q^{2} B_{1} W_{2} B_{1}^{T}-\Theta C^{T} V^{-1} C \Theta=0 \\
& K_{O}=\Theta C^{T} V^{-1}
\end{aligned}
$$

where the matrices $A_{g}$ and $B_{g}$ are given by Eq. (18) and Eq. (19).

$$
\begin{gathered}
A_{r}=\left[\begin{array}{ll}
0 & 1 \\
0 & 0
\end{array}\right] \\
B_{r}=\left[\begin{array}{ll}
1 & 0 \\
0 & 1
\end{array}\right]
\end{gathered}
$$

Moreover, after studying the characteristics of the noise and disturbances corrupting the electrical signals in today's cars [4-9,66,67], the process noise intensities $W_{1}=W_{2}=W$, the measurement noise intensity $V$, the state weighting matrix $Q$ and the control weighting matrix $R$ were chosen as given below (Eq. (20) - Eq.(23)).

$$
\begin{gathered}
W_{1}=W_{2}=W=\left[\begin{array}{lll}
1 & 0 & 0 \\
0 & 1 & 0 \\
0 & 0 & 1
\end{array}\right] \\
V=\left[\begin{array}{ll}
1 & 0 \\
0 & 1
\end{array}\right] \\
Q=\left[\begin{array}{cc}
1000 & 0 \\
0 & 1000
\end{array}\right] \\
R=\left[\begin{array}{ll}
1 & 0 \\
0 & 1
\end{array}\right]
\end{gathered}
$$

At this point, it is important to point out that $K_{C}$ is a two by three matrix consisting of the optimum deterministic controller $K_{L Q R}$ given by Eq. (15), which is obtained after solving for $\Pi$ in the algebraic Riccati equation (ARE) Eq. (14) [68-70], and a unitary gain vector. In addition, $K_{O}$ is given by Eq. (17) and is obtained after solving for $\Theta$ in the ARE Eq. (16), where the parameter $q$ is a scalar variable. Also, as $q$ approaches infinity, the loop transfer function recovers asymptotically its robustness properties. 
Furthermore, in accordance with the separation theorem, the optimization of performance in the presence of disturbances was carried out ignoring the noise, achieving an optimum deterministic controller whose gain matrix is given by Eq. (24).

Moreover, the optimization of the observer for estimating the state vector in the presence of white noise on the observation and white noise disturbances was carried out satisfactorily, achieving the Kalman filter gains given by Eq. (25) for $q$ equal to 100.

$$
\begin{aligned}
K_{\text {LQR }} & =\left[\begin{array}{ccc}
31.6188 & 0.4999 & 1 \\
0.4999 & 31.6346 & 1
\end{array}\right] \\
K_{O} & =\left[\begin{array}{cc}
1 & 0.9913 \\
0.9913 & 14.1071 \\
0.8687 & 99.9962
\end{array}\right]
\end{aligned}
$$

Therefore, after carrying out all the procedure explained in this section, the loop transfer function given by Eq. (26) - Eq. (31) (or Eq. (32) - Eq. (36), in state-space form) was achieved, and the singular values of the sensitivity function of the system (see Eq. (37)) are shown in Fig. 5. Note that for $q$ equal to 100 , a maximum singular value of approximately $2.41 \mathrm{~dB}$ (or 1.32) is achieved, which is considered a satisfactory result.

$$
L(s)=\frac{\left[\begin{array}{ll}
n 11(s) & n 12(s) \\
n 21(s) & n 22(s)
\end{array}\right]}{\operatorname{den}(s)}
$$

where

$$
\begin{aligned}
& n 11(s)=s^{5} 9.95 \cdot 10^{-14}+s^{4} 39.98+s^{3} 1386.88+s^{2} 49.99 \\
& n 12(s)=s^{5} 8.53 \cdot 10^{-14}+s^{4} 138.39+s^{3} 4739.38+s^{2} 4659.72+s 49.99 \\
& n 21(s)=s^{5} 1.42 \cdot 10^{-13}+s^{4} 32.79+s^{3} 966.01-s^{2} 1784.67 \\
& n 22(s)=s^{5} 8.53 \cdot 10^{-14}+s^{4} 546.76+s^{3} 20936.28+s^{2} 106939.83-s 1784.67 \\
& \operatorname{den}(s)=s^{5} 78.36+s^{4} 1490.44+s^{3} 109.38
\end{aligned}
$$

In addition, the loop transfer function in state-space form is given by Eq. (32) - Eq. (36).

$$
L(s)=\left[\begin{array}{l|l}
A l & B l \\
\hline C l & D l
\end{array}\right]
$$


where

$$
\begin{aligned}
& A l=\left[\begin{array}{cccccc}
-32.6188 & -0.4912 & -1 & 1 & 0.9913 & 0 \\
-1.4912 & -45.7417 & 0 & 0.9913 & 14.1071 & 0 \\
-0.8687 & -99.9962 & 0 & 0.8687 & 99.9962 & 0 \\
0 & 0 & 0 & 0 & 1 & 0 \\
0 & 0 & 0 & 0 & 0 & 1 \\
0 & 0 & 0 & 0 & 0 & 0
\end{array}\right] \\
& B l=\left[\begin{array}{ll}
0 & 0 \\
0 & 0 \\
0 & 0 \\
1 & 0 \\
0 & 1 \\
0 & 0
\end{array}\right] \\
& C l=\left[\begin{array}{llllll}
31.6188 & 0.4999 & 1 & 0 & 0 \\
0.4999 & 31.6346 & 1 & 0 & 0 & 0
\end{array}\right] \\
& D l=\left[\begin{array}{ll}
0 & 0 \\
0 & 0
\end{array}\right]
\end{aligned}
$$

Furthermore, the sensitivity function of the closed loop feedback system is given by Eq. (37)

$$
S(s)=\frac{I}{I+L(s)}
$$

where $I$ stands for the 2-by-2 identity matrix and $L(s)$ is the loop transfer function.

Basically, a trade-off was established between the noise rejection performance of the system and its robustness, resulting in the achievement of a satisfactory compromise between them by adjusting the parameter $q$. Such an adjustment process was carried out by increasing $q$ step-by-step from zero up to the value given above.

\section{The results of the experiment}

The system designed in this research was implemented as an intelligent system embedded in a car under performance tests. To that end, a laptop computer and the National Instruments Data Acquisition Card DAQCard-700 were used along with an analogue signal conditioning circuit consisting of a general-purpose operational amplifier TL084 and a few resistors and capacitors reasonably situated. In addition, the sampling frequency was $500 \mathrm{~Hz}$.

Fig. 6 shows the information coming from the wheel speed sensor, after being conditioned, and Fig. 7 shows the information coming from the accelerometer during one of the performance tests. During this test, the car was at approximately $84 \mathrm{~km} / \mathrm{h}$ when the driver hit on the brakes. Note that there is a high quantity of noise corrupting such information. 
The reality is that working with the information shown in Fig. 6 and Fig. 7 can lead to misleading conclusions. Such a corruption in the signals coming from both sensors justified the use of the robust estimator designed in this paper. Fig. 8 shows the power spectrum magnitude of the information coming from the sensors.

Consequently, Fig. 9 and Fig. 10 show the result of the robust estimation process of the true signal from the corrupted information shown in Fig. 6 and Fig. 7, respectively. Note that the system based on the LQG/LTR regulator has considerably diminished the noise corrupting the relevant signals while leaving them practically unchanged from the engineering standpoint.

Fig. 11 shows the power spectrum magnitude of the estimated speed and acceleration. It is important to point out the satisfactory reduction of the noise corrupting the important information.

In Fig. 7 and Fig. 10 the ringing (or damped oscillation) shown at the end of both figures is the effect of the vertical movement, the pitch and the roll, yielded by the car when the driver hit on the brakes. The eigenfrequencies of this ringing lay between $1 \mathrm{~Hz}$ and $2 \mathrm{~Hz}$. Their effects do not alter the experiment. Furthermore, the final acceleration read from the sensor should be zero because the car is stopped, but the accelerometer has a small offset that we usually cancel by using software techniques.

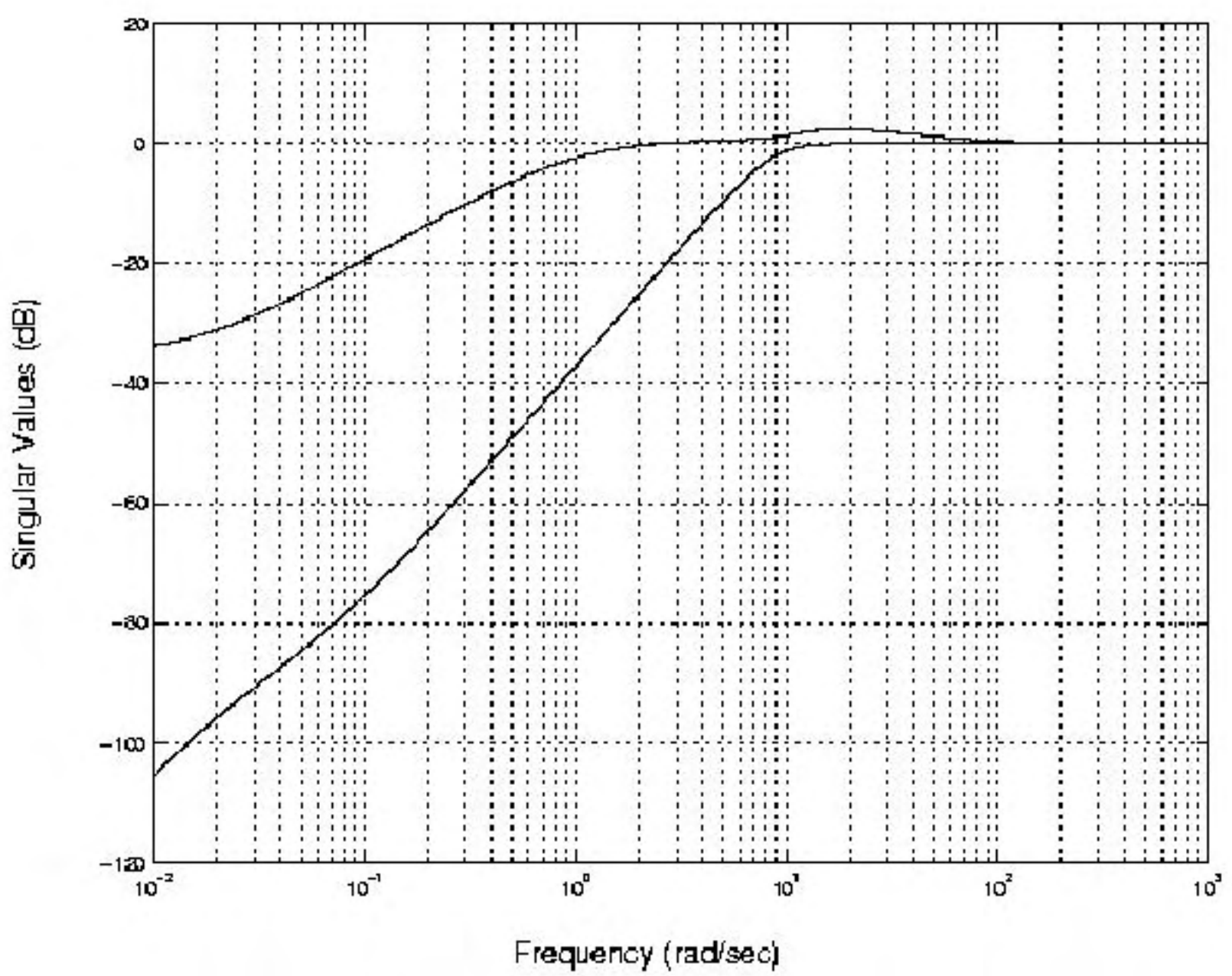

Figure 5. Singular values of the sensitivity function $(\mathrm{dB})$. 


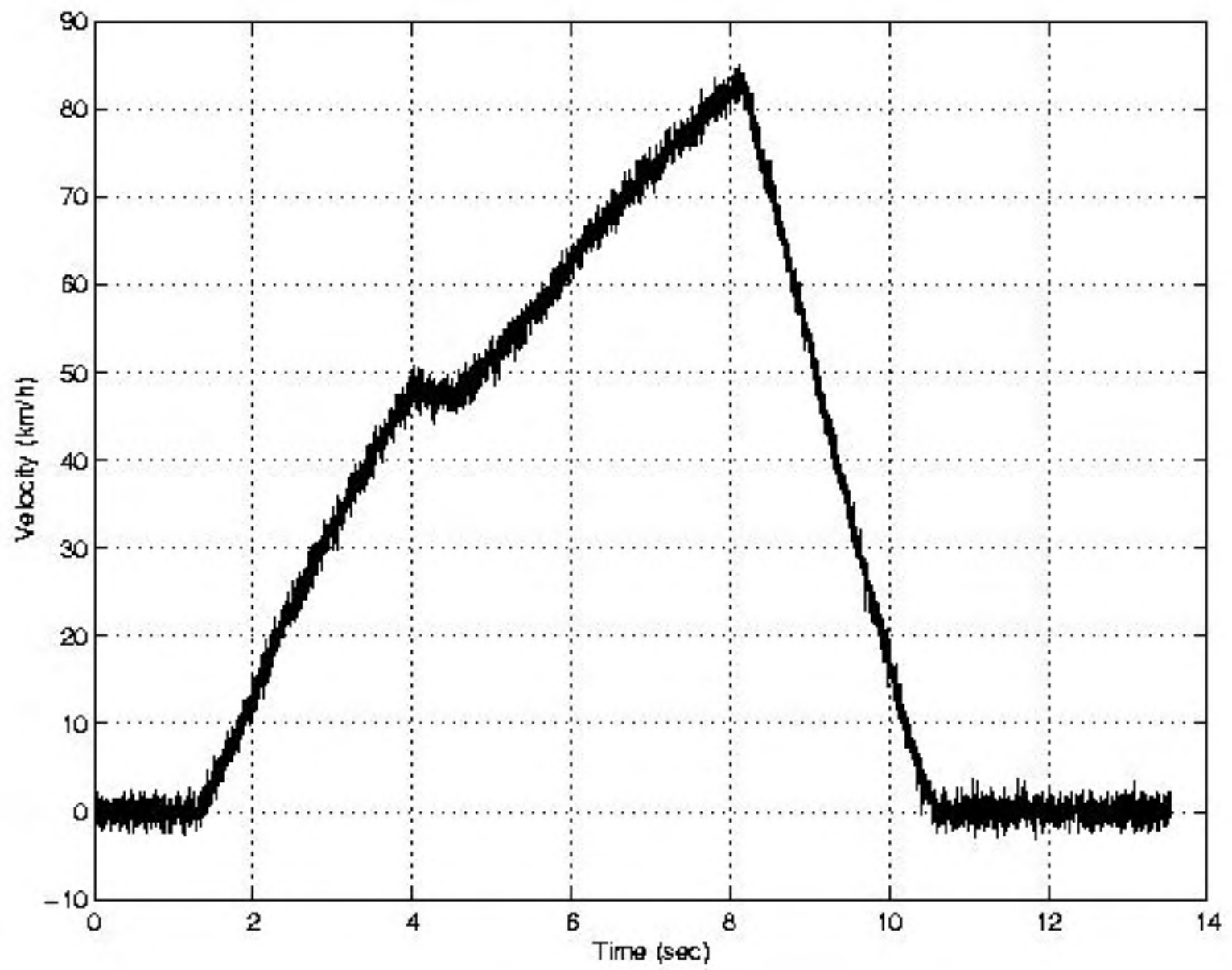

Figure 6. Velocity of the car (km/h) vs. Time (sec).

Before moving on to the conclusions, it is important to point out that the satisfactory results obtained in this paper made a comparison between this estimator and any other unnecessary. If the random processes are white and Gaussian, the optimum observer will be optimum under any reasonable performance criterion. However, according to Friedland [37], the theoretical framework hardly exists for the treatment of anything but white noise.

Even though the uncertainty in the parameters that define the dynamics of our process is not white noise, one practical way of dealing with this uncertainty is to assume that it is white noise, and the justification for this is the satisfactory improvement of the robustness of the resulting feedback controlled system. Furthermore, if we had used another estimator, the results of the experiment might not have been as good as the ones achieved in this paper.

However, another way to solve the problem of estimation presented is to carry out the estimation of the acceleration of the car under performance tests and its speed by using two independent single-input 
single-output optimal filters. Nevertheless, this solution implies using more electronic devices, which increases the cost of the system and the number of operations that the microprocessor embedded in the car has to carry out.

To sum up, despite there being several ways to carry out the estimation of the speed and the acceleration of a car under performance tests, the method presented here seems to be one of the most reliable, practical, inexpensive and efficient methods to estimate the above dynamic variables in today's cars.

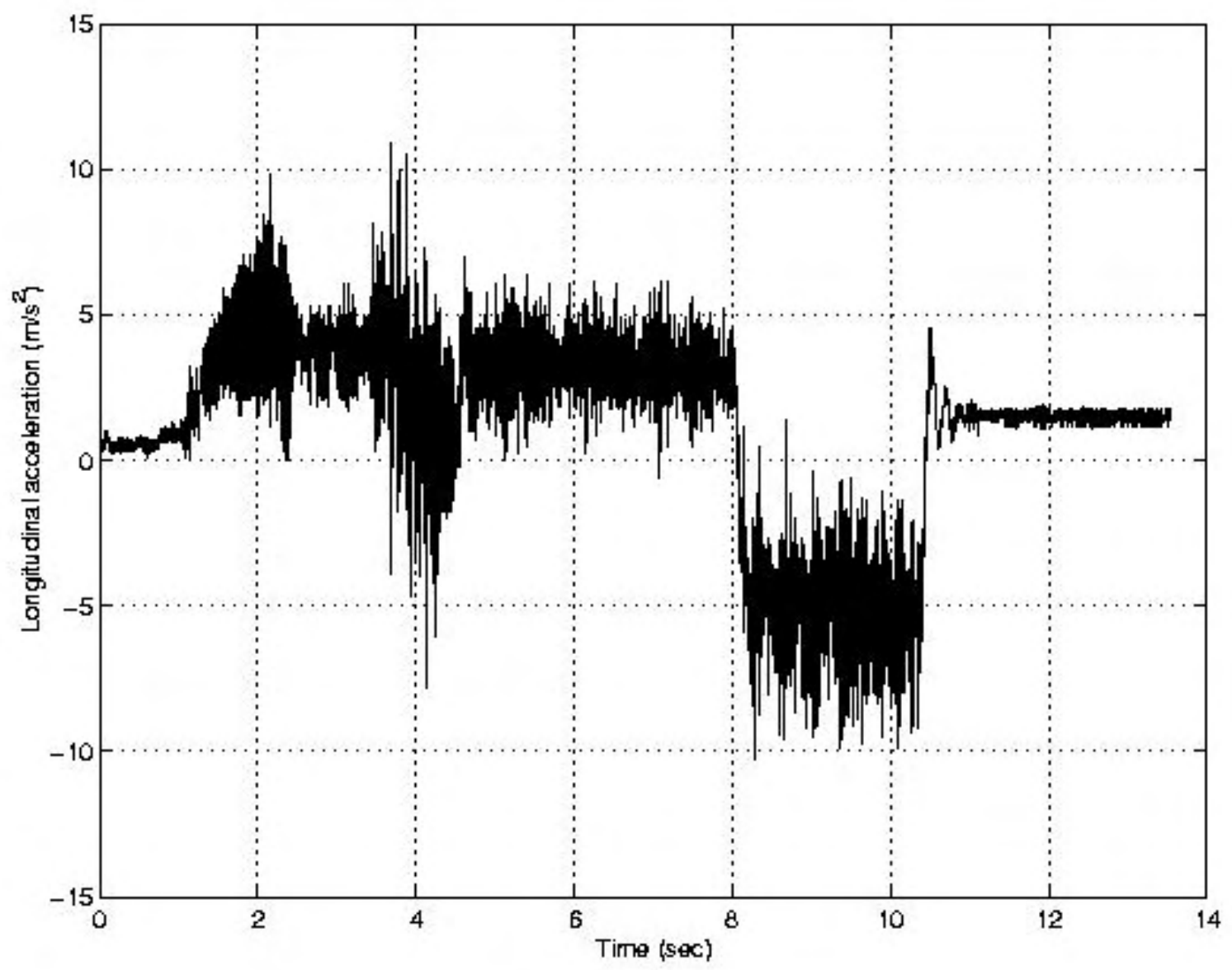

Figure 7. Longitudinal acceleration of the $\operatorname{car}\left(\mathrm{m} / \mathrm{s}^{2}\right)$ vs. Time $(\mathrm{sec})$. 

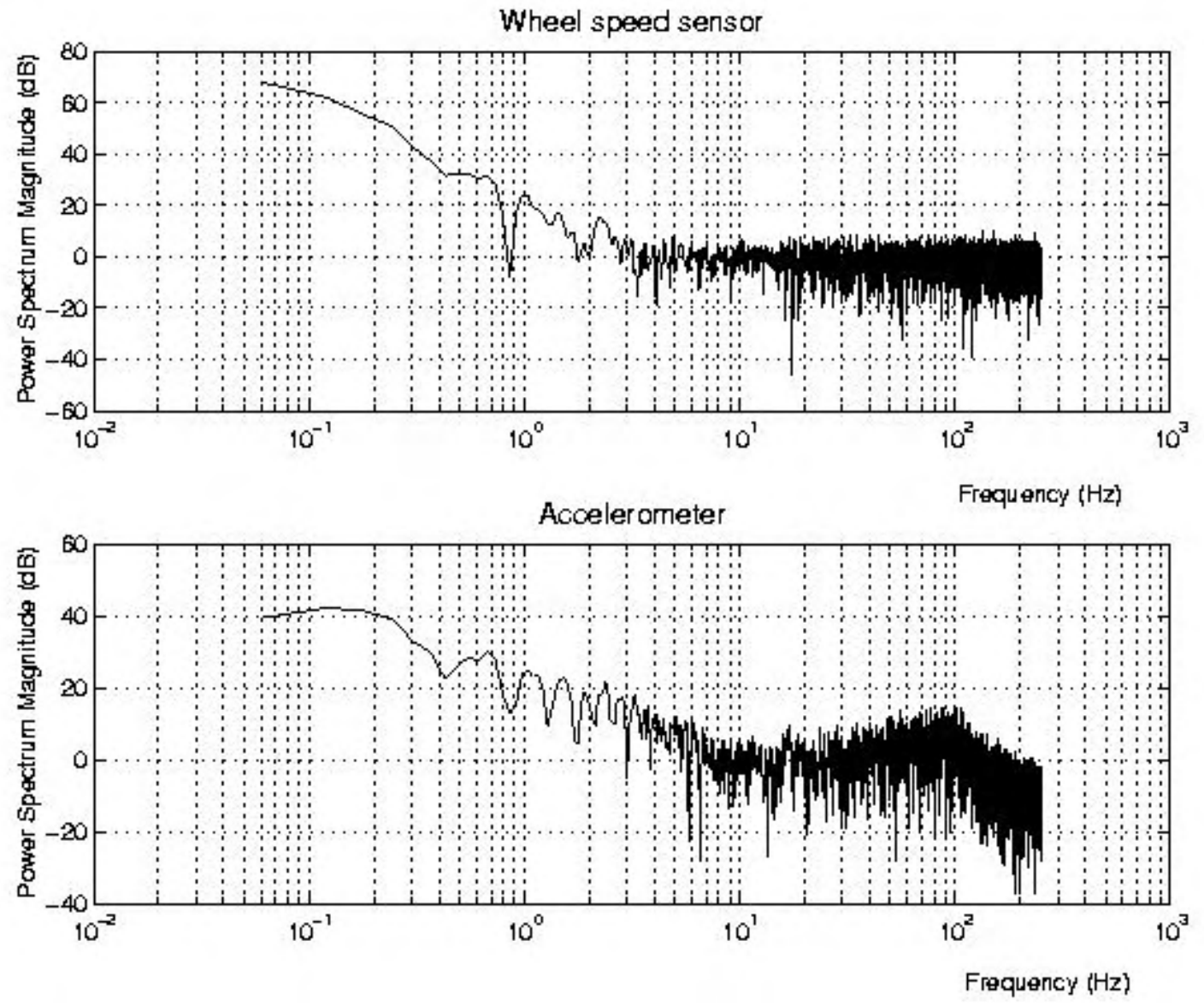

Figure 8. Power spectrum magnitude of the information coming from the wheel speed sensor and the accelerometer (dB). 


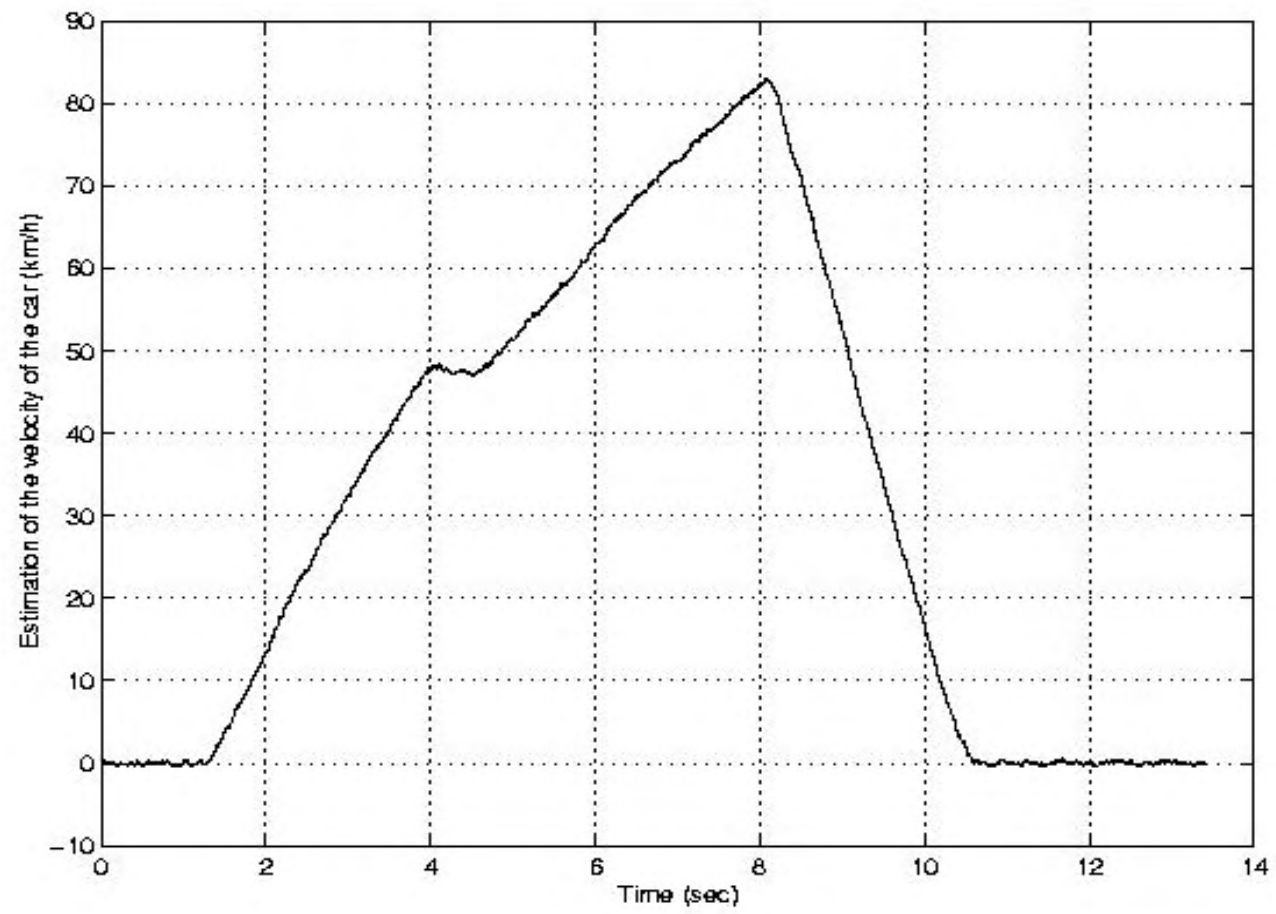

Figure 9. Estimation of the velocity of the car $(\mathrm{km} / \mathrm{h})$.

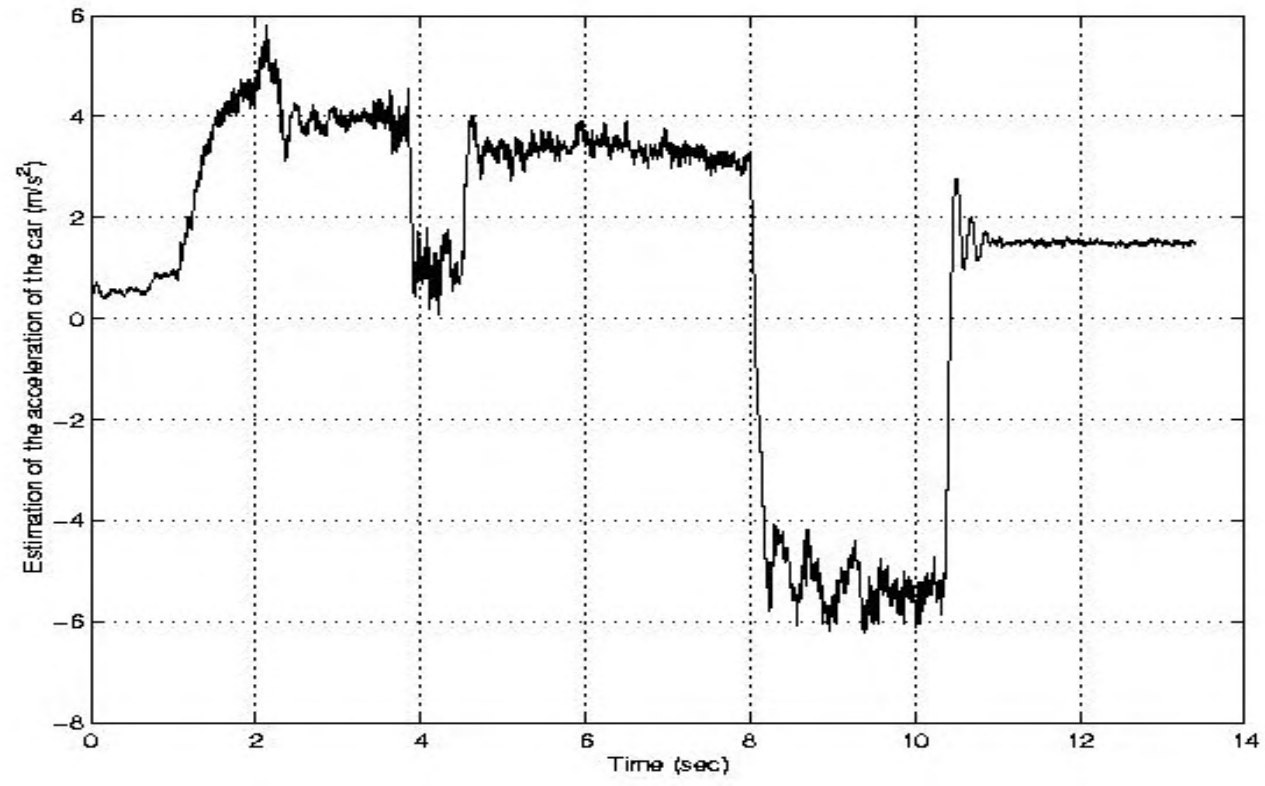

Figure 10. Estimation of the acceleration of the $\operatorname{car}\left(\mathrm{m} / \mathrm{s}^{2}\right)$. 

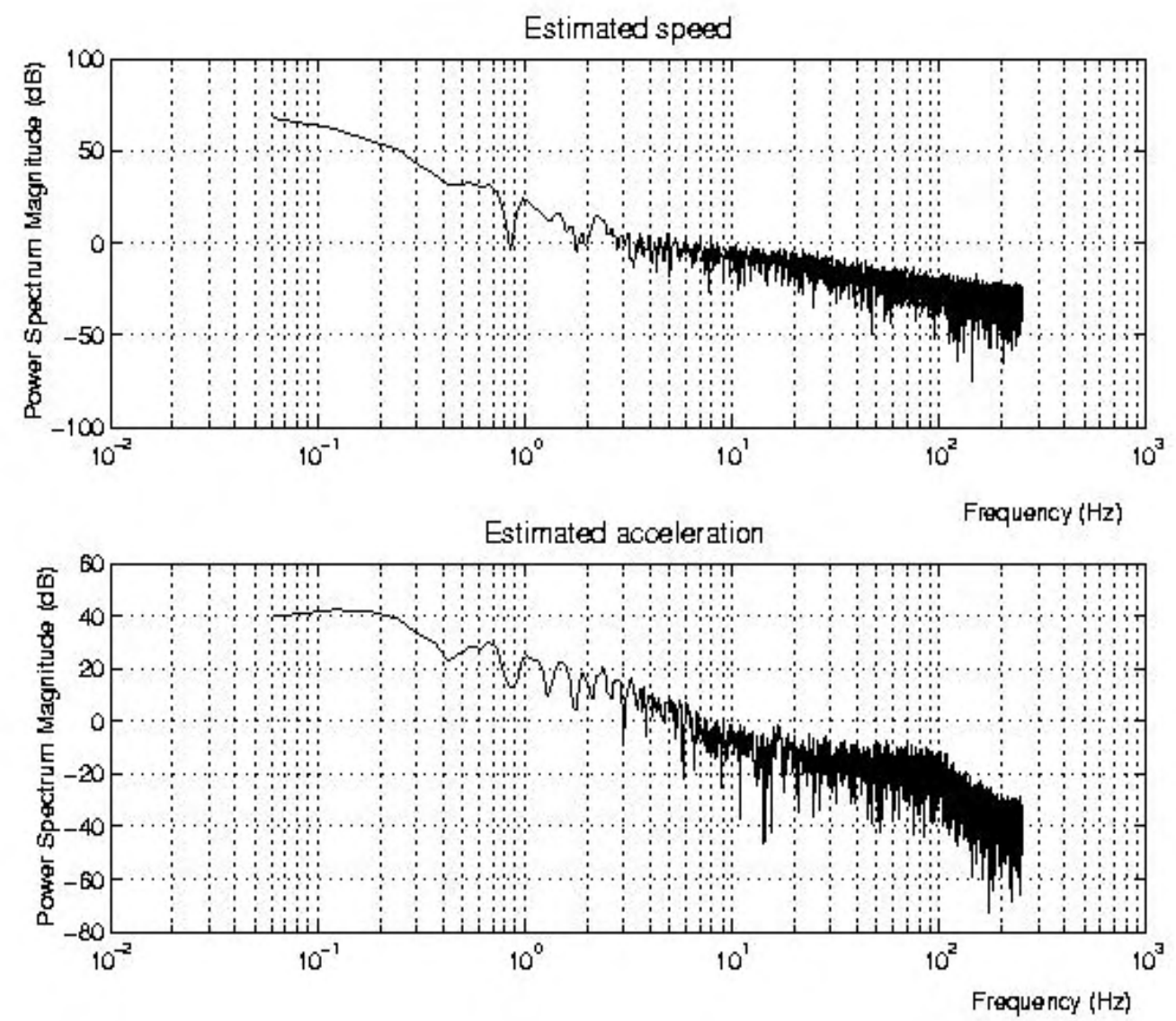

Figure 11. Power spectrum magnitude of the estimated speed and the estimated acceleration (dB).

\section{Conclusions}

To conclude, in this paper a robust and optimal estimator for a multi-input multi-output sensor system was designed and tested under laboratory conditions. Here, the noise corrupting the electrical information coming from both a wheel speed sensor of a car under performance tests and its acceleration was diminished by using robust estimation techniques.

In addition, the two-input two-output dynamic system and the optimum observer were shaped in the sense that the system was robust against modeled and unmodeled uncertainties. For this reason, the robust estimator designed here can work satisfactorily in environments where the working conditions are severe, for example, in the automotive industry.

The results show that despite the signals coming from real physical systems are corrupted by noise and interferences, and that the behavior of such systems is affected negatively by undesirable disturbances, the use of robust and optimal control techniques can bring satisfactory results.

Furthermore, it is important to stress that the system designed in this paper is easy to implement and was built by using low-cost components, which makes the use of this technology affordable for car manufacturers. 
Moreover, as the loop transfer function resulting from the method presented was shaped to deal with models whose parameters have a certain degree of uncertainty, the system does not need expensive sensors to work satisfactorily. Actually, the cost of the electronic devices is a very important factor to be taken into consideration when building non-luxury cars.

Last but not least, the use of this paper's design method can bridge the gap between intelligent control methods and the design of sensors and actuators for a wide range of applications. The reality is that only by the fusion of these concepts can the designer find the way clear to build the intelligent sensors that today's cars need.

\section{Acknowledgements}

This work has been supported by the Department of Circuits and Systems in the EUIT de Telecomunicación, School of Telecommunication Engineering at the Universidad Politécnica de Madrid, Madrid, Spain.

\section{References}

1. Hernandez, W. Fluxgate magnetometer for magnetic fields in the range 1-100mT. IEE Electronics Letters 1995, 31, 2110-2111.

2. Hernandez, W. Magnetic-field sensor based on a relaxation oscillator. Sensors and Actuators A 1996, 55, 163-166.

3. Rodriguez, F.; Trujillo, H.; Hernandez, W. A simple bandgap type magnetoamplifier. Sensors and Actuators A 1996, 55, 133-137.

4. Hernandez, W. Improving the response of an accelerometer by using optimal filtering. Sensors and Actuators A 2001, 88, 198-208.

5. Hernandez, W. Improving the response of several accelerometers used in a car under performance tests by using Kalman filtering. Sensors 2001, 1, 38-52.

6. Hernandez, W. Improving the response of a wheel speed sensors by using an adaptive line enhancer. Measurements 2003, 33, 229-240.

7. Hernandez, W. Improving the response of a wheel speed sensors by using frequency-domain adaptive filtering. IEEE Sensors Journal 2003, 3, 404-413.

8. Hernandez, W. Improving the response of wheel speed sensors by using robust and optimal signal processing techniques. IEEE International Symposium on Industrial Electronics, ISIE2005, Dubrovnik, Croatia, June 20-23, 2005, 1049-1053.

9. Hernandez, W. Wheel speed sensors. In Encyclopedia of Sensors; Grimes, C. A., Dickey, E. C., Pishko, M. V., Ed.; American Scientific Publishers, in press.

10. Dean, M. Semiconductor and Conventional Strain Gages; Academic Press, 1962.

11. Canfield, E. B. Electromechanical Control Systems and Devices; Wiley, 1965.

12. Keast, D. N. Measurements in Mechanical Dynamics; McGraw-Hill, 1968.

13. Farago, F. T. Handbook of Dimensional Measurements; Industrial Press, 1968.

14. Norton, H. N. Handbook of Transducers for Electronic Measuring Systems; Prentice-Hall, 1970.

15. Norton, H. N. Sensor and Analyzer Handbook; Prentice-Hall, 1982. 
16. Herceg, E. E. Handbook of Measurement and Control; Schaevitz Engineering, Camden, New Jersey, 1972.

17. Mansfield, P. H. Electrical Transducers for Industrial Measurement; Butterworths, 1973.

18. Fertis, D. G. Dynamics and Vibration of Structures; Wiley-Interscience, 1973.

19. Meirovitch, L. Elements of Vibration Analysis; McGraw-Hill, 1975.

20. Gregory, B. A. Introduction to Electrical Instrumentation and Measurement Systems; Macmillan, 1981.

21. Beckwith, T. G.; Buck, N. L.; Marangoni, R. D. Mechanical Measurements, Third Edition; Addison-Wesley, 1982.

22. Beckwith, T. G.; Marangoni, R. D.; Lienhard, J. H. Mechanical Measurements, Fifth Edition; Addison-Wesley, 1993.

23. Carr, J. J. Elements of Electronic Instrumentation and Measurements, Third Edition; PrenticeHall, 1995.

24. Johnson, C. D. Process Control Instrumentation Technology, Fifth Edition; Prentice-Hall, 1997.

25. Edgar, P. Sensors for Measurement and Control; Addison-Wesley Longman, 1998.

26. Robert Bosch GmbH (Hrsg.), Kraftfahrtechnisches Taschenbuch, 25 ${ }^{\text {th }}$ Ed.; Vieweg Verlag, 2004.

27. Fleming, W. J. Overview of automotive sensors. IEEE Sensors Journal 2001, 1, 296-308.

28. Kwakernaak, H. Optimal low-sensitivity linear feedback systems. Automatica 1969, 5, 279-285.

29. Athans, M. The role and use of stochastic linear-quadratic gaussian problem in control system design. IEEE Transactions on Automatic Control 1971, 16, 529-552.

30. Kwakernaak, H.; Sivan, R. Linear Optimal Control Systems; Wiley-Interscience, 1972.

31. Safonov, M. G.; Athans, M. Gain and phase margin of multiloop LQG regulators. IEEE Transactions on Automatic Control 1977, 22, 173-178.

32. Doyle, J. C. Guaranteed margins for LQG regulators. IEEE Transactions on Automatic Control 1978, 23, 756-757.

33. Doyle, J. C.; Stein, G. Robustness with observers. IEEE Transactions on Automatic Control 1979, 24, 607-611.

34. Safonov, M. G. Stability and Robustness of Multivariable Feedback Systems; MIT Press, 1980.

35. Safonov, M. G.; Laub, A. J.; Hartman, G. Feedback properties of multivariable systems: The role and use of return difference matrix. IEEE Transactions on Automatic Control 1981, 26, 47-65.

36. Doyle, J. C.; Stein, G. Multivariable feedback design: Concepts for a classical/modern synthesis. IEEE Transactions on Automatic Control 1981, 26, 4-16.

37. Friedland, B. Control System Design; McGraw-Hill, 1986.

38. Francis, B. A Course in $H_{\infty}$ Control Theory; Springer-Verlag, 1987.

39. Maciejowski, J. M. Multivariable Feedback Design; Addison-Wesley, 1989.

40. Anderson, B. D.; Moore, J. B. Optimal Control: Linear Quadratic Methods; Prentice-Hall, 1989.

41. Ackerman, J.; Bartlett, A.; Kaesbauer, D.; Sienel, W. Robust Control: Systems with Uncertain Plant Parameters; Springer, 1993. 
42. Engelbert, H. J.; Karatzas, I.; Rockner, M. Stochastic Processes and Optimal Control; Gordon \& Breach Science Publications, 1993.

43. Stengel, R. F. Optimal Control and Estimation; Dover Publications, 1994.

44. Pavel, N. H. Optimal Control of Differential Equations; Marcel Dekker, 1994.

45. Lewis, F. L; Syrmos, V. L. Optimal Control, $2^{\text {nd }}$ Edition, Wiley-Interscience, 1995.

46. Basar, T.; Bernhard, P. $H^{\infty}$ Optimal Control and Related Minimax Design Problems: A Dynamic Game Approach, $2^{\text {nd }}$ Edition, Birkhauser, 1995.

47. Saberi, A.; Sannuti, A.; Chen, B. M. $H_{2}$ Optimal Control; Prentice-Hall, 1995.

48. Whittle, P. Optimal Control: Basics and Beyond; John Wiley \& Sons, 1996.

49. Skoghestad, S.; Postlethwaite, I. Multivariable Feedback Control; John Wiley \& Sons, 1996.

50. Zhou, K.; Doyle, J. C.; Glover, K. Robust and Optimal Control; Prentice-Hall, 1996.

51. Hager, W. W.; Pardalos, P. M. Optimal Control: Theory, Algorithms and Applications; Springer, 1998.

52. Burl, J. B. Linear Optimal Control; Prentice-Hall, 1998.

53. Vinter, R. Optimal Control; Birkhauser, 2000.

54. Locatelli, A. Optimal Control: An Introduction; Birkhauser, 2001.

55. Naidu, D. S. Optimal Control Systems; CRC Press, 2002.

56. Ackerman, J. Robust Control: The Parameter Space Approach, $2^{\text {nd }}$ Edition, Springer, 2002.

57. Bryson, A. E. Applied Linear Optimal Control: Examples and Algorithms; Cambridge University Press, 2002.

58. Burghes, D.; Graham, A. Control Theory and Optimal Control; Albion/Horwood Publications, 2003.

59. Hull, G. D. Optimal Control Theory for Applications; Springer, 2003.

60. Kirk, D. E. Optimal Control Theory: An Introduction; Dover Publications, 2004.

61. Doob, J. L. Stochastic Processes; John Wiley \& Sons, 1953.

62. Dynkin, E. B. Markov Processes; Springer-Verlag, 1965.

63. Papoulis, A. Probability, Random Variables, and Stochastic Processes, Fourth Edition, McGraw-Hill, 2001.

64. Hernandez, W. Robust Control of DC-DC Switching Converters and Low Distortion Audio Power Amplifiers, Ph.D. dissertation, Department of Electronics, Universitat Ramón Llull, Barcelona, Spain, 1999.

65. Hernandez, W.; Mayer, A. Robust frequency compensation of a low distortion audio power amplifier based on the listener perception, International Symposium on Quantitative Feedback Theory and Frequency Domain Methods, Durban, South Africa, August 26 - 27, 1999, 75-78.

66. Hernandez, W.; Mayer, A.; Alvarez, A. Robust control applied to a dc-dc step-down switching regulators for 14/42V hybrid architectures. FISITA World Automotive Congress, FISITA2004, Barcelona, Spain, May 23-27, 2004.

67. Hernandez, W.; Mayer,A.; Alvarez, A. $\mathrm{H}_{\infty}$ control of a step-down dc-dc switching regulator for $14 / 42 \mathrm{~V}$ hybrid architectures of next-generation cars. $30^{\text {th }}$ Annual Conference of the IEEE Industrial Electronics Society, IECON 2004, Busan, Korea, November 2-6, 2004, 442-447.

68. Kalman, R. E. Contributions to the theory of optimal control. Proc. 1959 Mexico City Conference on Differential Equations, Mexico City, Mexico, 1960, 102-199. 
69. Kleinman, D. L. On iterative technique for Riccati equation computation. IEEE Transactions on Automatic Control 1968, 3, 114-115.

70. Lasiecka, L.; Triggiani, R. Differential and Algebraic Riccati Equations with Applications to Boundary /Point Control Problems: Continuous Theory and Approximation Theory, Lecture Notes in Control and Information Science, vol. 164, Springer-Verlag, 1991.

(C) 2005 by MDPI (http://www.mdpi.org). Reproduction is permitted for noncommercial purposes. 